\title{
What Physics Drives the Unified Model?
}

\author{
Michael A. Dopita \\ Mt. Stromlo $\mathcal{B}$ Siding Spring Observatory, Institute of Advanced \\ Studies, The Australian National University, Australia
}

\begin{abstract}
The Unified Model holds that the aspect-dependent effects primarily determine the apperance of the active galactic nucleus that we observe. However, additional parameters will be needed to fully unify the different tribes of AGN. Three parameters; aspect, accretion rate into the nuclear regions, and the evolutionary status of the central black hole probably hold the key to "grand" unification schemes.
\end{abstract}

\section{What is the Unified Model?}

The Black Hole (BH) paradigm of Active Galactic Nuclei (AGN) is now universally accepted, and explains the structure of AGN in terms of an accretion disk which may or may not include a relativistic jet emerging in the poleward direction. The "unified model" was developed to clarify the relationship between the various sub-classes of AGN (Rowan-Robinson 1977; Lawrence and Elvis 1982; Antonucci and Miller 1985; Lawrence 1987) and has been summarised in two excellent reviews (Antonucci 1993 and Urry and Padovani 1995) which are essential reading. The basic idea behind the unified model is that orientation effects exercise a profound influence on the appearance of the AGN, and determine the sub-class into which it will be categorised. The "strong" unification hypothesis; that all AGN classes are fundamentally the same phenomenon seen at different orientations, has not proven successful. However, in its more restricted form; that some classes of object transform into other classes as a function of viewing angle, it seems secure. The cause of the orientation effect is believed to be a geometrically thick and optically-thick dusty molecular torus located about the equatorial plane of the AGN which may obscure it from direct view.

A second orientation effect is found in the radio-loud categories, for which the unification concept was orginally developed (Orr \& Browne 1982; Barthel 1991). AGN which are observed close to the polar direction display apparently super-luminal motions, rapid variability, intense power-law continua, flat radio spectra and high linear polarisation all due to relativistic beaming effects associated with the jet. The BL Lac objects, and the optically-violently variable (OVV) or flat-spectrum radio quasars (FSRQs) all seem to fit into this category (Blandford and Rees, 1978; Blandford and Konigl 1979). Combining these two effects, the following transformations apply. Seyfert $1 \Leftrightarrow$ Seyfert 2; QSOs $\Leftrightarrow$ Ultraluminous IRAS Narrow-line Galaxies; BL Lac / Blazar $\Leftrightarrow$ Double lobe radio Elliptical Galaxy.While these are satisfying as far as they go, there is still no way to fully unify the various tribes of AGN. In particular, there is no explana- 
tion of the radio-loud : radio-quiet dichotomy. Clearly, a parameter other than orientation must play a role. It has been variously argued that radio loudness is related to host galaxy type (Smith et al. 1986), black hole spin (Blandford 1990; Wilson and Colbert 1995), or to differences in the rate of nuclear feeding (Rees et al. 1982; Baum, Zirbel and O'Dea 1995).

\section{How Can the Dusty Torus Exist?}

The dusty torus is usually depicted as a fat doughnut surrounding, or forming the outer edge of, the inner thin accretion disk. Such a configuration is, of course, not dynamically consistent with a steady state, since if it is composed of a continuous gaseous medium, it will collapse to a thin disk on a timescale of order half the orbital timescale. Nor, as pointed out by Krolik \& Begelman (1988), can it be supported by internal gas pressure, since, in order for it to be geometrically thick, it would have to have a temperature of order $10^{6} \mathrm{~K}$. Any dust present would then be very rapidly destroyed. Physically self-consistent models for a thick torus depend upon the existence of an accretion flow towards the central engine.

According to Krolik \& Begelman (1988) the dusty gas is confined into a large number of self-gravitating clouds in dynamic balance between mergers and tidal shearing. Cloud-cloud collisions drive a net inward flow. The mean column density of the clouds in this model is very large $\left(\sim 10^{24} \mathrm{~cm}^{2}\right)$. However this seems incompatible with obervations of rotational support of the dense regions of the accretion flow (Jackson et al. 1993), and the relatively low line-of-sight column densities $\left(\sim 10^{23} \mathrm{~cm}^{2}\right)$ inferred for the thick torus (Dopita et al. 1998). The X-ray absorption region must be located closer to the central engine.

The alternative picture, that the thick torus simply represents a large-scale accretion flow of a continuous medium toward the nucleus, is more compatible with observation. In this model, the inner boundary of the torus is determined by the dust sublimation point. Near the mid-plane the rotationally-supported gas will fall almost perpendicular to the accretion disk, and is brought to rest by passage of the gas through a symmetric pair of accretion shocks. Thus, such a structure produces a dense, geometrically thin accretion disk embedded in the mid-plane, and extending within, the inner edge of the thick torus.

\section{What is the Role of Accretion?}

Merger events act on the gas through torques and dissipation to dump a large fraction $\left(\sim 10^{10} M_{\odot}\right)$ into the nuclear regions $(e . g$. Solomon, Downes and Radford 1992). These processes have been amply confirmed by theoretical calculations (Barnes and Hernquist 1991, 1992, 1996). At the point where orbital support of the gas becomes important (within $300 \mathrm{pc}$ of the centre) a strong accretion shock is formed, which is likely to trigger a major nuclear starburst within a dynamical timescale of $\sim 10^{8}$ years.

Matter dumped into the central kiloparsec of a galaxy has still to lose a great deal of angular momentum before it can be fed down into the AGN. However, star formation itself provides an efficient means of re-distributing the angular momentum. See, for example, Collin (this volume), or Bekki (1995). If these 
processes work down to small enough scales within the accretion disk, then we may expect a symbiosis between the amount of gas fed down to the AGN, and the amount of gas converted to stars in the accretion flow. This may provide a physical basis for the Kormendy relationship between the stellar bulge mass, and the $\mathrm{BH}$ mass, which seems to apply to both $\mathrm{E}$ and $\mathrm{S}$ galaxies.

As far as the unified model is concerned, the appearance of the AGN should depend critically upon the rate of accretion into the nuclear regions. For subEddington accretion we would obtain the classic Shakura and Sunyaev (1973) thin disk, and there should be no optically-thick accretion torus. Such disks allow the free escape of the relativistic jet from the nuclear region, but are ineffective in producing either a broad-line or a extended narrow line emission region. The radio jets in such systems are characterised by efficient relativistic boosting, and can therefore give rise to Blazar phenomena. The sub-Eddington (and possibly, advective) nature of the accretion will ensure that such sources are of relatively low luminosity, and of low radio power. Such objects might be identifiable with the FR I radio sources as postulated in the unified models of radio sources (Urry and Padovani 1995).

At very high (super-Eddington) accretion rates into the nuclear regions, the accumulation of dusty matter in the nuclear regions tends to obscure the central engine. This may be identified with the dusty "thick" torus of the unified models. Indeed, Dopita et al. (1998) have demonstrated that if the thick dusty accretion torus is an optically thick accretion flow, it must be highly superEddington. If the flow of gas into the broad-line region is also super-Eddington, much of the matter entering these regions must be lost in the form of a thermal or magnetic wind (e.g. Chiang and Murray, 1996; Lovelace, this volume). At high enough mass-loss rates, this wind will become optically-thick to electron scattering, providing a hot electron-scattering photosphere to reprocess the hard radiation (EUV, $\mathrm{X}$ - and $\gamma$-rays) from the central $\mathrm{BH}$ and the innermost portions of the accretion disk. This would serve to obscure the central engine from direct view except in the polar directions where the relativistic jets may escape. Such a toroidal reprocessing photosphere would also provide enough absorption to explain the weakness of Seyfert 2 galaxies in X-rays (Mushotzky, Done \& Pounds 1993), and its inner surface could also be used for K- $\alpha$ scattering. In this model, an AGN seen in an intermediate angle (but outside the "thick" dusty torus) would appear as either a QSO or as a Sy 1 galaxy, depending on the BH mass.

Models with a thermal radiation-driven wind allow the possibility of direct interaction between the thermal and relativistic winds. This interaction is favoured when the accretion into the BLR is highly super-Eddington with respect to the nuclear $\mathrm{BH}$. When such interaction occurs below the reprocessing photosphere, it will lead to mass entrainment into the jet, and a slowing of the jet to highly sub-relativistic speeds. This is likely to be the cause of radio-loud: radio-quiet dichotomy, as discussed below. Consider a simple (toy) parametric form for the mass accretion rate into the nuclear core region;

$$
\dot{M}(t)=\dot{M}_{a v} \exp \left[-t / \tau_{a c c}\right] \times\left(1-\exp \left[-t / \tau_{a c c}\right]\right)
$$

with only one characteristic timescale, the accretion timescale:

$$
\tau_{a c c}=\lambda \tau_{f f}=\lambda R^{3 / 2} /(G M)^{1 / 2}
$$


For $R \approx 10 \mathrm{kpc}$ and $M \approx 10^{10} M_{\odot}, \tau_{a c c} \approx 1.5 \times 10^{8} \lambda$ years. The accretion rate is super-Eddington provided:

$$
\dot{M}_{B H}=M_{B H} / \tau_{B H}
$$

where $\tau_{B H}$ is the growth timescale for the $\mathrm{BH}$ accreting at the Eddington limit. This can be calculated from the luminosity:

$$
L_{B H}=\phi \dot{M}_{B H} c^{2}=\theta L_{e d d}=\theta 4 \pi c G M_{B H} / \kappa
$$

where $\phi$ is the fraction of the rest mass energy radiated by matter falling into the BH. For a Schwartzschild BH $\phi=\left[1-\left(2^{3 / 2} / 3\right)\right]$. Typically $\phi \sim 0.1$. The factor $\theta$ is the fraction of the Eddington Luminosity produced by this accretion, and is assumed $\sim 1$. Thus, the growth timescale of the $\mathrm{BH}$ is:

$$
\tau_{B H}=M_{B H} / \dot{M}_{B H}=\frac{\kappa c}{4 \pi G}\left(\frac{\phi}{\theta}\right) \approx 4.4 \times 10^{8}\left(\frac{\phi}{\theta}\right) \text { years }
$$

inserting numerical values, $\tau_{B H} \approx 5.10^{7}$ years. Thus, the growth timescale of the $\mathrm{BH}$ is of the same order than, or somewhat smaller than $\tau_{a c c}$, so that appreciable growth of the $\mathrm{BH}$ ( from $10^{5-7} M_{\odot}$ to $10^{8-9} M_{\odot}$ ) can occur during a merging/dynamical collapse event. During this event, molecular clouds are presumably being converted rapidly to stars in a massive nuclear starburst, so that the growth of the $\mathrm{BH}$ is determined by the competition between inflow, accretion, and star formation.

\section{An Accretion Disk Photosphere?}

During super-Eddington accretion and possibly even later, the radiation pressure from the central $\mathrm{BH}$ would be sufficient to drive a thermal or magnetic wind. If this wind is accelerated by radiation pressure of the central source, the detailed theory has already been developed in a series of papers by Murray and his collaborators (Murray and Chiang, 1995; Murray et al. 1995; Chiang and Murray, 1996). For sufficient outflow rates the radiatively driven wind will be both dense, and optically thick to the escape of $\mathrm{X}$ - and $\gamma$ - ray photons from the central source so that the continuum observed is produced by a reprocessing photosphere dominated by electron scattering opacity. In this case, the momentum flux in the wind is given by:

$$
\dot{M}_{w} v_{w}=\left(\frac{\eta}{c}\right)\left(\frac{\Omega}{4 \pi}\right) L_{B H}
$$

where $\Omega$ is the solid angle covered by the optically-thick radiatively-driven wind, subtended at the $\mathrm{BH}$, and $\eta$ is the effective number of scatterings per photon. In stars, this factor is greater than unity; typically $\eta \sim 3-4$; however, in WolfRayet stars it may rise even higher. In such radiatively-driven winds, the outflow velocity is similar to the escape velocity at the base of the outflow. In the AGN case the wind originates at the inner edge of the region of super-Eddington flow, and has a velocity of order $3 \times 10^{4} \mathrm{~km} \mathrm{~s}^{-1}$. The photospheric radius occurs when the electron scattering optical depth is of order unity: 


$$
r_{p h o t}=\kappa\left(\frac{\Omega}{4 \pi}\right)^{-1}\left(\frac{\dot{M}_{w}}{4 \pi v_{w}}\right) \sim 1.0 M_{7} \text { light days }
$$

where $\kappa$ is the electron scattering opacity. This is consistent with variability studies and reverberation mapping analysis of nearby Sy 1 galaxies (Maoz, 1994). The effective temperature of the electron-scattering photosphere is given by Stefan's Law, with the radiation density diluted by a factor $\Psi \sim 10$ with respect to a Black-Body emitter;

$$
T=\left(\frac{4 \pi c^{2} \Psi}{\sigma \kappa^{2} \eta^{2}}\right)^{1 / 4} v_{w} L_{B H}^{-1 / 4} \sim 37000 K\left[\frac{\Psi}{10}\right]^{1 / 4}\left[\frac{\eta}{3}\right]^{-1 / 2} v_{4} L_{45}^{-1 / 4}
$$

Thus, when the wind velocity is of order $20,000 \mathrm{~km} \mathrm{~s}^{-1}$ the effective photospheric temperature is high enough $\left(\sim 10^{5} \mathrm{~K}\right)$ to provide a "big blue bump" in the continuum spectrum which, thanks to the high outflow velocity and the electron scattering, should provide only weak and broad photospheric lines. Such electron scattering dominated extended atmospheres will show only a weak Lyman Limit discontinuity. The spectral distribution of such an electron scattering photosphere is not characterised by a simple temperature. In general, such atmospheres give a power-law below the peak in emergent flux corresponding to the temperature given by eqn. (8), and roll off sharply above this peak. The power-law slope depends on the effective curvature of the atmosphere, i.e. on the ratio $\dot{M}_{w} / v_{w}$ (Abbott \& Conti, 1987). AGN atmospheres should have little curvature, giving a rather flat power-law at lower energies.

This photosphere is extended on both sides of the accretion disk, and illuminates and photoionises the surface layers or the infalling matter, giving rise to the broad-line region. The effective source temperature decreases for higher $\mathrm{BH}$ luminosities, providing lower ionisation conditions in the more luminous AGN. This is presumably the explanation of the Baldwin Effect in QSOs (Baldwin 1977, Baldwin et al. 1978, Kinney, Rivolo and Koratkar 1990). The wind equations imply that:

$$
L_{B H}=\frac{4 \pi \epsilon c G M_{B H}}{\kappa \eta}=\left(\frac{\epsilon}{\eta}\right) L_{E d d}
$$

Consistency therefore requires $\epsilon \sim \eta$ if the BH luminosity is to be of the same order as the Eddington Limit. The mechanical energy flux in the wind is simply related to the bolometric luminosity of the central object:

$$
L_{M e c h}=\left(\frac{\eta v_{w}}{2 c}\right)\left(\frac{\Omega}{4 \pi}\right) L_{B H}=0.05\left[\frac{\eta}{3}\right]\left[\frac{\Omega}{4 \pi}\right] v_{4} L_{B H}
$$

Finally, we can relate the mass flux in the wind to the mass flux onto the BH:

$$
\left(\frac{\dot{M}_{w}}{\dot{M}_{B H}}\right)=\left(\frac{\eta \phi c}{v_{w}}\right)\left(\frac{\Omega}{4 \pi}\right)=9\left[\frac{\phi}{0.1}\right]\left[\frac{\eta}{3}\right]\left[\frac{\Omega}{4 \pi}\right] v_{4}^{-1}
$$

Thus, the mass flux into the radiatively driven wind will dominate over the mass flux into the BH until the accretion disk becomes thin $(\Omega / 4 \pi \sim 0.1)$. 


\section{The Jet Model}

Let us adopt the jet-black hole symbiosis model of Falke and Biermann (1995) and Falke, Malkan and Biermann (1995):

$$
L_{j e t}=\gamma_{j e t} \dot{M}_{j e t} c^{2} \approx 0.3 L_{B H} ; \quad 3 \leq \gamma_{j e t} \leq 10
$$

It therefore follows immediately that :

$$
\left(\frac{\dot{M}_{j e t}}{\dot{M}_{B H}}\right)=\left(\frac{\phi}{3 \gamma_{j e t}}\right)
$$

Thus, the mass flux in the jet is only about $1 \%$ of the mass flux into the $\mathrm{BH}$, and more importantly, is only about $0.1 \%$ of the mass flux in the radiatively-driven wind, in the super-Eddington regime. This implies that any mixing between the jet and the thermal wind will be critical in determining whether the jet can escape or is mixed with thermal matter within the radio photosphere of the wind (determined by its radio free-free opacity). In the first case the object will be radio-loud - this is the case usually considered. In the second case, the very luminous synchrotron emission, and the synchrotron Compton losses all occur below the photosphere, and what emerges is a fast thermal wind (seen in some objects as a Broad Absorption Line QSO) which carries the energy flux to excite the narrow-line emission. This has only a small synchrotron component and produces the faint radio-emitting bubbles seen in radio-quiet objects such as Seyferts (Bicknell et al. 1998). Bicknell, Dopita and O'Dea (1997) have shown that the jet energy flux is related to the synchrotron power at frequency $\nu$ through an efficiency factor:

$$
\kappa_{v}=\left(\frac{5-\delta}{8-\delta}\right) f\left(t, \gamma_{0}, B, \nu, \alpha\right)=c E_{0}^{2 \alpha} F_{e} B^{(\alpha+1)} \nu^{-\alpha} t
$$

where $\delta$ is the index of the power law in the density distribution of the galactic medium $(\sim 2), t$ is the age of the source, $B$ the magnetic field in the radio lobes, $E_{0}$ is the lower energy limit of the relativistic electrons, $\alpha$ is the spectral index, and $F_{e}$ is the fraction of the internal energy of the plasma contained in relativistic electrons. In the super-Eddington phase, mixing with the thermal wind gives $F_{e} \sim 10^{-3}$. On the other hand, highly sub-Eddington accretion allows for the free escape of the relativistic plasma so $F_{e} \sim 1$. However, radio-quiet objects have lobe luminosities which are typically weaker by a factor of a thousand than their radio-loud counterparts. Thus, for a given jet energy flux, this difference in the entrainment factor is in itself sufficient to explain the difference between the radio-quiet, and the radio-loud cases, respectively, provided that the entrainment has occurred below a radio photosphere.

The transition between the radio-quiet and radio-loud cases presumably comes about at the time that the accretion rate into the BLR decreases to the point that the thermal wind starts to clear in the polar regions and entrainment into the radio jet is no longer enough to slow this to transonic speeds. This allows for transition objects displaying both BLRs and relativistic jets (radio loud QSOs). 


\section{What is the Role of Evolution and Aspect?}

In the case of a major merger event between two massive gas-rich systems, the initial phase should be a galaxy-wide starburst producing a luminous infrared galaxy with HII-like characteristics. In the later phases, gas flow toward the nucleus feeds the $\mathrm{BH}$ and outflow could then excite the Seyfert 2 - like emission seen in some of the very distant ultraluminous IRAS galaxies (Rowan-Robinson, et al. 1991, 1993; van Ojik 1994). Such mergers will initially be radio-quiet, since the supply of gas into the nuclear regions is sufficient to choke off highluminosity radio jets, but as nuclear accretion rates fall, radio jets might later escape to produce a high $\mathrm{z}$ radio source with its strong narrow-line emission region (Meisenheimer, Hippelein \& Neeser 1994; McCarthy, Spinrad and van Breugel 1995). Such mergers of gas-rich systems would have been much more common in the early universe and especially at the epoch of formation of the Abell clusters. Since this epoch was so early, $\mathrm{z} \sim 2-4$, the merging gas-rich systems may not even have had time to form regular spiral galaxies. However, let us call them 'spirals' for convenience. It is therefore reasonable to propose the following evolutionary scenario for 'primary' merger events giving rise to the QSOs and the majority of elliptical galaxies.:

$$
\begin{aligned}
\mathrm{Sp}+\mathrm{Sp} & \Rightarrow \text { Starburst } \\
& \Rightarrow \text { Postmerger + RQQSO } \\
& \Rightarrow \mathrm{E}+\text { massive BH+radio jets (?) }
\end{aligned}
$$

In the event of a later merger event:

$$
\begin{aligned}
\mathrm{E}+\mathrm{Irr} & \Rightarrow \text { Dust-lane E + Nuclear starburst } \\
& \Rightarrow \text { Dust-lane E + GPS radio source } \\
& \Rightarrow \text { Dust-lane E + FR II radio source } \\
& \Rightarrow \text { Dust-lane E + FR I radio source }
\end{aligned}
$$

Alternatively, the case of M87 suggests that low-power radio sources may be fed by a subsequent accretion episode at sub-Eddington rates, such as feeding through a cooling flow:

$$
\mathrm{E}+\text { Cooling flow } \Rightarrow \mathrm{E}+\mathrm{LINER}+\mathrm{FRI} \text { or } \mathrm{E}+\text { Blazar }
$$

Thus, evolution determines the nature of the AGN, but aspect determines the type of AGN we see from outside.

\section{Conclusions}

The arguments given here (for an extended account, see Dopita 1997), establish the feasibility of the hypothesis that galactic mergers determine, as much as does orientation, the nature and evolutionary stage of the AGN that we observe. Such mergers also produce a massive star burst, linking them with luminous and 
ultra-luminous infrared galaxies. The size of the BHs produced depend on the total gas supply. For much of the early growth phases, the supply of gas into the region of influence of the $\mathrm{BH}$ is likely to be super-Eddington. This is capable of producing an optically-thick radiatively-driven wind, which may choke off the relativistic jets in this phase, producing a radio-quiet AGN.

For sub-Eddington accretion rates, such as found in either elliptical mergers or in cooling flows, the radio jets can freely escape from the thin accretion disk, so radio-loud AGN and relativistically-boosted BL Lac /Blazer phenomena will be preferentially produced in this case.

Acknowledgments. I wish to thank Ski Antonucci, Geoff Bicknell, Stefi Baum, Anuradha Koratkar, Ralph Sutherland, Pete McGregor, Charlene Heisler, Agris Kalnajs, Mark Whittle and Wil van Breugel for many stimulating conversations and physical insights in the preparation of this work. I also wish to thank the Australian Dept. of Industry, Science and Tourism (DIST) for support under an IS\&T major grant.

\section{References}

Abbott, D. C., \& Conti, P. S. 1987, Ann. Rev. A \& A, 25, 113.

Antonucci, R. 1993, Ann. Rev. A\& A, 31, 473

Antonucci, R. \& Miller, J.S. 1985, ApJ, 297, 621.

Baldwin, J. A. 1977, ApJ, 214, 679.

Baldwin, J. A., Wampler, E. J., \& Gaskell, C. M. 1978, Nature, 273, 431.

Barthel, P. D. 1991, Advances in Space Research, 11, 231.

Barnes, J. E., \& Hernquist, L. 1991, ApJ, 370, 65.

Barnes, J. E., \& Hernquist, L. 1992, Ann. Rev. A\&A, 30, 705.

Barnes, J. E., \& Hernquist, L. 1996, ApJ, 471, 115.

Baum, S. A., Zirbel, E. I. \& O'Dea, C. P. 1995, ApJ, 451, 88.

Bekki, K. 1995, MNRAS, 276, 9.

Bicknell, G.V, Dopita, M.A. \& O’Dea, C. 1997, ApJ 490,202.

Bicknell, G.V et al. 1998, ApJ, 495, 680.

Blandford 1990, in Active Galactic Nuclei, eds. T. J.-L. \& M. Mayor Saas-Fee Advanced Course 20, (Berlin: Springer), p161.

Blandford, R.D. \& Rees, M. J. 1978, in Pittsburgh Conference on BL Lac Objects, ed. A. N. Wolfe (Pittsburgh: U.Pittsburg Press), p328.

Blandford, R. D. \& Konigl, A. 1979, ApJ, 232, 34.

Chiang, J. \& Murray, N. 1996, ApJ, 466, 704.

Dopita, M. A. 1997, PASA, 14, 230.

Dopita, M. A., et al. 1998, ApJ, 498, 570.

Falcke, H., \& Biermann, P.L. 1995, A\&A., 293, 665..

Falcke, H., Malkan, M.A. \& Biermann, P.L. 1995, A\&A., 298, 375.

Kinney, A. L., Rivolo, A. R., \& Koratkar, A. P. 1990, ApJ, 357, 338.

Krolik, J.H. \& Begelman, M.C. 1988 ApJ, 329,702. 
Lawrence, A. 1987, PASP, 99, 309.

Lawrence, A. \& Elvis, M. 1982, ApJ, 256, 410.

McCarthy, P. J., Spinrad, H., \& van Breugel, W. 1995, ApJ, 447, 77.

Maoz, D. 1994, in Reverberation Mapping of the Broad-Line Region in AGN, eds. P. M. Gondhalekar, K. Horne, \& B. M. Peterson, ASP Conf. Ser, 69,95 .

Meisenheimer, K., Hippelein, H. \& Neeser, M. 1994, The First Stromlo Symposium : The Physics of Active Galaxies, eds. G. V. Bicknell, M. A. Dopita \& P. J. Quinn, ASP Conf. Ser., 54, 397

Murray, N., \& Chiang, J. 1995, ApJ, 459, L105.

Murray, N. et al. 1995, ApJ, 451, 498.

Mushotzky, R. F., Done, C., \& Pounds, K. A. 1993, Ann. Rev. A\&A, 31, 717.

Orr, M. J. L., \& Browne, I. W. A. 1982, MNRAS, 200, 1067.

Rees, M. et al. 1982, Nature, 295, 17.

Rowan-Robinson, M. 1977, ApJ, 213, 638.

Rowan-Robinson, M. et al. 1991, Nature, 351, 719.

Rowan-Robinson, M. et al. 1993, MNRAS, 261, 513.

Shakura, N. I., \& Sunyaev, R. A. 1973, A\&A, 24, 337.

Smith, E.P. et al. 1986, ApJ, 306, 64.

Urry C.M, \& Padovani, P. 1995, PASP, 107, 803.

van Ojik, R., et al. 1994, A\&A, 289, 54.

Wilson, A. S., \& Colbert, E. J. M. 1995, ApJ, 438, 62. 